\title{
Song pattern recognition in the grasshopper Chorthippus biguttulus: the mechanism of syllable onset and offset detection
}

Accepted: 12 March 2001 / Published online: 4 May 2001

(C) Springer-Verlag 2001

\begin{abstract}
The male song of the duetting grasshopper Chorthippus biguttulus consists of syllables alternating with noisy pauses. The syllable-pause structure is important for song recognition by the female. Using playback experiments we investigated the mechanism by which intensity modulations within the song pattern are used to detect syllable onsets and offsets. We varied the relative onset level (level of the syllable beginning relative to the noisy pause) and the relative offset level (level of the noisy pause relative to the syllable end) independently in different experiments. For all females, an increase in intensity defining the syllable onset was necessary to evoke responses. Syllable offset cues were not always necessary: some females responded to continuous noise stimuli wherein only syllable onsets were marked by short pulses of high intensity. Those females that did not require syllable offset cues did not, however, lack a functional pause detection mechanism, since their responses to model songs containing silent pauses were restricted to a given range of pause durations. We propose that syllable-pause detection involves two independent processes: (1) syllable onset detection by a phasic neuronal unit that can be re-activated only after a short pause, and (2) the rejection of unacceptably long pauses by a second unit.
\end{abstract}

Keywords Acoustic communication · Pattern recognition - Pause detection - Auditory interneuons · Chorthippus biguttulus (Acrididae)

\author{
R. Balakrishnan \\ Centre for Ecological Sciences, Indian Institute of Science, \\ Bangalore 560012, India \\ E-mail: rohini@ces.iisc.ernet.in \\ D. von Helversen \\ Max-Plank-Institut für Verhaltensphysiologie, \\ 82319 Seewiesen, Germany \\ E-mail: helversen@mpi-seewiesen.mpg.de \\ O. von Helversen \\ Institut für Zoologie II, Universität Erlangen, \\ Staudtstrasse 5, 91058 Erlangen, Germany
}

\section{Introduction}

Chorthippus biguttulus is an acoustically communicating gomphocerine grasshopper in which pair formation is achieved by duetting between the sexes. In common with a number of other acoustically communicating insect species (Cade 1985; Alexander et al. 1997; Pollack 1998), Ch. biguttulus females use song pattern as a cue for species recognition and mate choice (von Helversen and von Helversen 1994). The song of the male Ch. biguttulus consists of verses 2-3 s in length (Fig. 1). Each verse has a distinctive syllable-pause structure, with syllable durations ranging from about $40-100 \mathrm{~ms}$ and pause durations from 8-25 ms, depending on the individual and on the temperature of stridulation (von Helversen 1972; von Helversen and von Helversen 1997). As in other gomphocerine grasshoppers, stridulation in Ch. biguttulus occurs by rubbing a row of pegs on the inner side of the hind femur against a specialized vein on the forewing. Each peg strike results in the production of a very brief click (of 250-450 $\mu$ s duration) or impact of sound (Fig. 1), and a verse of Ch. biguttulus is thus composed of a series of impacts produced at different rates depending on the velocity of movement. A syllable of Ch. biguttulus is produced by three successive down-up strokes, with the two hind legs moving slightly out of phase (Elsner 1974, 1983), which causes a camouflage of the brief gaps at the reversal points of movement. Typically, the first 10-12 ms of the syllable (produced by the first down-stroke) is higher in intensity than the rest. Each syllable is followed by a short period (10-15 ms at $30^{\circ} \mathrm{C}$ ) of lower intensity, which we refer to as a noisy pause. The lower intensity during the pause results from the fact that, during this period, only one of the hind legs (usually the lagging one) continues to move and engage the pegs against the wing vein at a lower rate than during the syllable (Fig. 1; Elsner 1974).

Over the past several years, the duetting response of the female has been used in playback experiments to elucidate many features of the song pattern recognition 


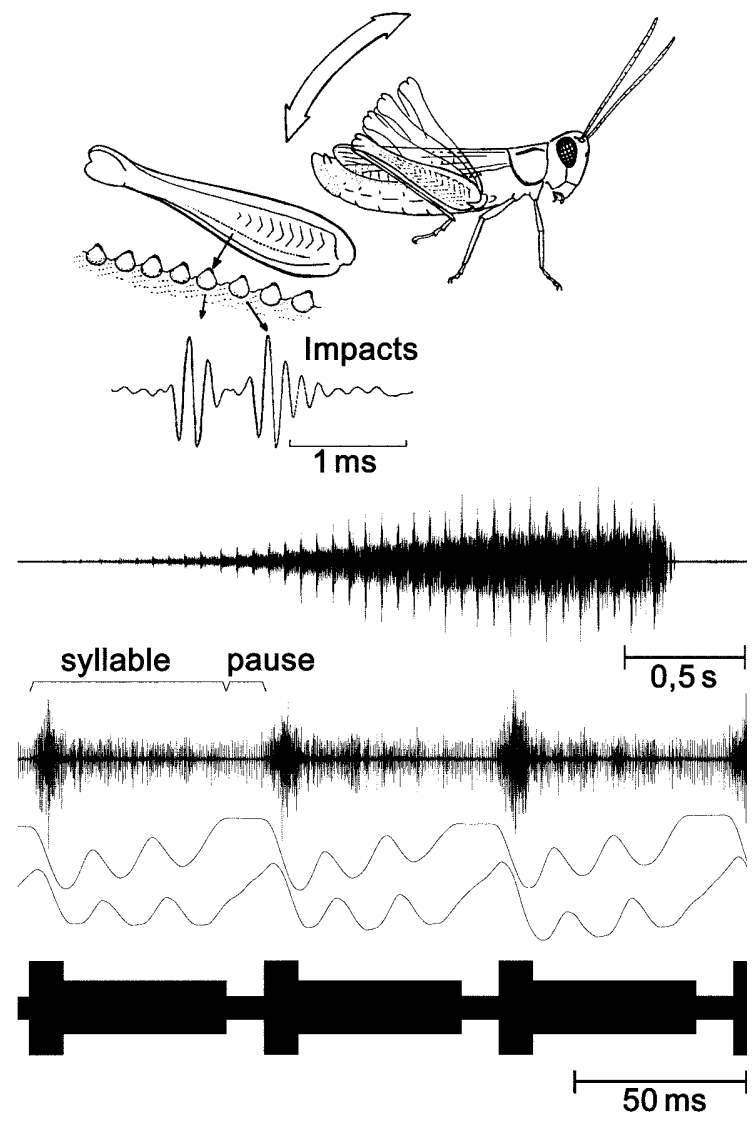

Fig. 1 The male song of Chorthippus biguttulus. Above: stridulation occurs by rubbing a row of pegs on the inner side of the hind femur against a modified wing vein. Each peg strike results in the production of a short sound impulse or impact $(250-450 \mu$ s in duration). Below: the top trace shows an oscillogram of a verse of male song. A magnified view of a section of the verse is shown in the second trace to illustrate the syllables and noisy pauses. The third and fourth traces show simultaneous recordings of the movements of the two legs using an opto-electronic device (von Helversen and Elsner 1977). Downward deflections represent downstrokes, upward deflections depict upstrokes and horizontal sections represent no movement. Note that the two legs are out of phase and that the lagging leg continues to move at a lower rate during the pauses. The bottom trace is a schematic representation of the stimuli used, in which two parameters were varied: sound level during the pause and the accentuated beginning of a syllable, while the intensity of the rest of the syllable remained constant

mechanism in this species (von Helversen and von Helversen 1994). The experiments were performed using synthetic model songs, typically consisting of whitenoise syllables separated by silent pauses. On the basis of these extensive studies, we now know that Ch. biguttulus females use a number of features for song recognition. These include (1) the presence of both low- $(6-8 \mathrm{kHz})$ and high- $(20-30 \mathrm{kHz})$ frequency components in the song spectrum, (2) a minimum syllable duration of about $40 \mathrm{~ms}$, and (3) an optimum syllable-pause duration ratio of about 5:1 (von Helversen 1972; von Helversen and von Helversen 1997). In Ch. biguttulus, there is no sharp tuning to the species-specific syllable repetition rate or syllable duration per se (von Helversen and von Helversen 1983, 1998). This is in interesting contrast to other insect song recognition systems, which are often tuned to conspecific syllable repetition rates and durations (Weber and Thorson 1989; Hennig and Weber 1997; Schul 1998). Thus, song recognition in Ch. biguttulus does not seem to occur on the basis of band-pass filtering for syllable repetition rates, as appears to be the case in crickets (Schildberger 1984; Weber and Thorson 1989; Hennig and Weber 1997), nor can it be explained in terms of cross-correlation with an internal template (von Helversen and von Helversen 1998). The evidence so far suggests a mechanism that measures successive syllable and pause durations in the time domain.

The measurement of syllable and pause durations would require the detection of syllable onsets and offsets. Syllable-pause discrimination is based on differences in intensity between syllables and pauses rather than on changes in impact rate or spectral cues ( $R$. Balakrishnan et al., unpublished observations). In this context, we investigated the role of intensity differences between syllables and pauses in the processes of syllable onset and offset detection. We examined the effect on female response by varying the relative onset level (sound level of the syllable beginning relative to the noisy pause) and the relative offset level (sound level of the noisy pause relative to the syllable end) in independent experiments, using artificial sound stimuli of the type shown in Fig. 1 (bottom trace).

\section{Materials and methods}

\section{Animals}

Adult Ch. biguttulus males and last instar female larvae were collected in the field during July-October of 1997-1999 in Bavaria, Germany and the northern Alps. Females were collected as larvae to ensure virginity. Males and females were housed in separate cages $(50 \mathrm{~cm} \times 50 \mathrm{~cm} \times 80 \mathrm{~cm})$ and fed on fresh grass (Dactylis glomerata). The animals were on a $14: 10 \mathrm{~h}$ light:dark cycle and maintained at a temperature between $25^{\circ} \mathrm{C}$ and $30^{\circ} \mathrm{C}$. Cages were monitored every day and every freshly moulted adult female was individually marked with a three-point colour code. Thus, the age of every female was known to within 1 day at the time of testing. Females were tested between 4 days and 14 days of the last moult.

\section{Song models}

Song models were synthesised using either the Turbolab (Bressner Technology) signal-processing program or with custom-built software. All song models consisted of segments (syllables) of white noise $(80 \mathrm{~ms}$ in duration) recorded from a random noise generator and then digitised at $200 \mathrm{kHz}$. Depending on the experiment, the pauses (12 ms in duration) were either noisy or silent. In most experiments, noisy pauses contained impacts at a rate of $1 \mathrm{kHz}$ (the modal value in pauses of natural songs). In some experiments, broad-band noise was used instead of impacts in the pauses since, for a given relative sound level between syllable and pause, we found no difference in response between stimuli containing noise versus impacts in the pauses. Individual impacts in the pauses of model songs were synthesised by addition of three sine waves $(7 \mathrm{kHz}+22 \mathrm{kHz}+30 \mathrm{kHz})$, with the relative input amplitudes adjusted such that the loudspeaker outputs yielded impacts of short durations $(300-400 \mu \mathrm{s})$ and broad-frequency spectrum. 

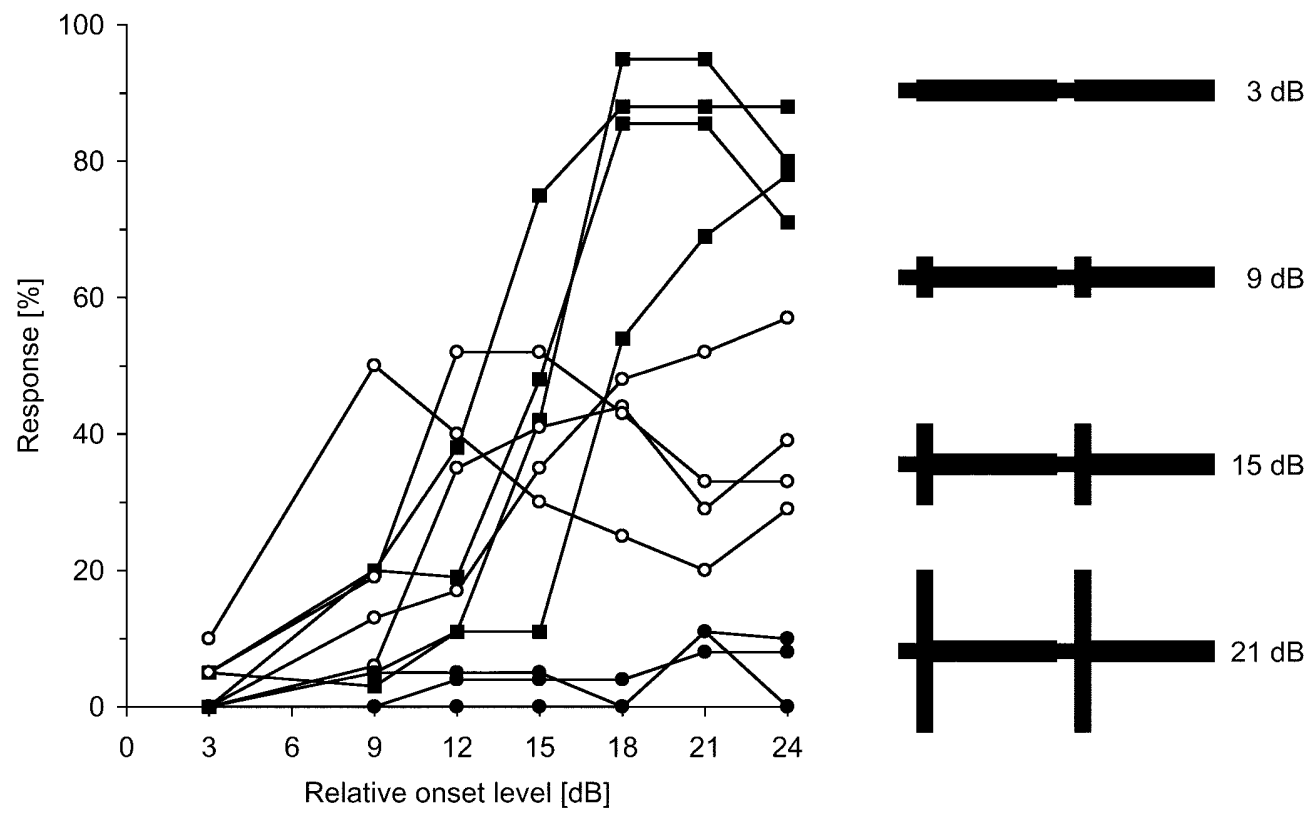

Fig. 2 The effect of varying the relative onset level. The graph shows the results of playback experiments in which the amplitude of the first $10 \mathrm{~ms}$ of each syllable of a model song (80-ms noise syllables, 12-ms pauses) was systematically increased (see stimulus series on the right) over that in the pauses (the first increase was a step of $6 \mathrm{~dB}$, the subsequent steps being of $3 \mathrm{~dB}$ each). Each line represents the response profile of one female ( $n=11$ females), measured in all experiments as the percent response to each song model (20-35 presentations of each model). The symbols represent females which showed a very low response (filled circles), females with medium response level (open circles), and females responding at a high level (squares)

When constructing a series of impacts within the pauses, the successive impact periods were randomly jittered around a value of $1 \mathrm{~ms}$ (for a 1-ms separation of impacts, any individual impact period had a value between $0.95 \mathrm{~ms}$ and $1.05 \mathrm{~ms}$ ). This jittering was intended to obtain an output sound frequency spectrum that was broad-band and continuous (Michelsen et al. 1985). A verse of model song thus consisted of around 33 iterations of a single 'syllable ( $80 \mathrm{~ms}$ ) plus pause (12 ms)' segment and was typically $3 \mathrm{~s}$ long. The standard 80/12 stimulus, which was played back in every experiment, consisted of a 3-s-long verse comprised of rectangular white noise syllables (of $80 \mathrm{~ms}$ duration) separated by silent pauses (12 ms). The relative onset level was varied by accentuation of the first $10 \mathrm{~ms}$ of the syllable, whereas the relative offset level was varied by attenuation of the amplitude of the noise/impacts in the pauses.

\section{Playback experiments}

The synthesised stimuli were played back using custom-built power amplifiers and Dynaudio (D21/2) dome tweeter loudspeakers (flat frequency response: $2-40 \mathrm{kHz}$ ). The syllable intensity of every stimulus was calibrated to the desired level of playback in a soundattenuated chamber using a Bruel and Kjaer Sound Level Meter (Type 2231). In addition, the loudspeaker outputs of most stimuli were recorded using a Bruel and Kjaer condenser microphone (1/2 inch) and Sound Level Meter (Type 2231) on a Racal tape recorder $(2-40 \mathrm{kHz})$, digitised and analysed using Turbolab software to confirm the relative intensity levels between syllables and pauses. Stimuli were played back at $64 \mathrm{~dB}$ SPL (RMS, for the nonamplitude-modulated part of the noise syllables).

Virgin females were placed in a small gauze cage in a soundattenuated chamber $\left(30 \pm 1^{\circ} \mathrm{C}\right)$ and a computer-controlled set-up automatically played back verses of song at the desired rate (with

a minimum interval of $30 \mathrm{~s}$ ) and also recorded any female reply that might occur in between. Responsive females were selected by placing a singing male (confined in a small gauze cage) into the female cage and choosing a female that gave consistent replies to the male calling song. Approximately $70 \%$ of females selected in this manner remained motivated enough to duet through a given testing session (which often lasted 5-12 h). A single testing session typically consisted of 20-35 cycles, where each cycle involved playback of 25-30 different model song verses in a random order (i.e. 20-35 presentations of each song model). Females were also tested with a 3 -s-long continuous white noise stimulus and only those that responded with less than $5 \%$ probability to white noise were selected for the analysis (less than $5 \%$ of females tested responded in a non-specific manner to white noise). The second criterion used was that only females showing a response higher than $30 \%$ to at least one of the stimuli presented in a given experiment were considered for the analysis (about $20 \%$ of all females tested did not fulfil this criterion). The percentage response to each song model was then calculated for each female.

\section{Results}

In order to investigate the role of intensity modulation between syllables and pauses in pattern recognition, three features were varied during the course of these experiments: (1) relative onset level defined as the sound level of the first $10 \mathrm{~ms}$ of the syllable relative to the preceding noisy pause, (2) relative offset level defined as the sound level of the noisy pause relative to the end of the preceding syllable, and (3) syllable onset accentuation, defined as the sound level of the first $10 \mathrm{~ms}$ of the syllable with respect to the rest of the syllable (see Fig. 1).

\section{Syllable onset cues are necessary}

The accentuated intensity at the beginning of each syllable commonly found in Ch. biguttulus male songs may serve as an unambiguous marker that allows detection 

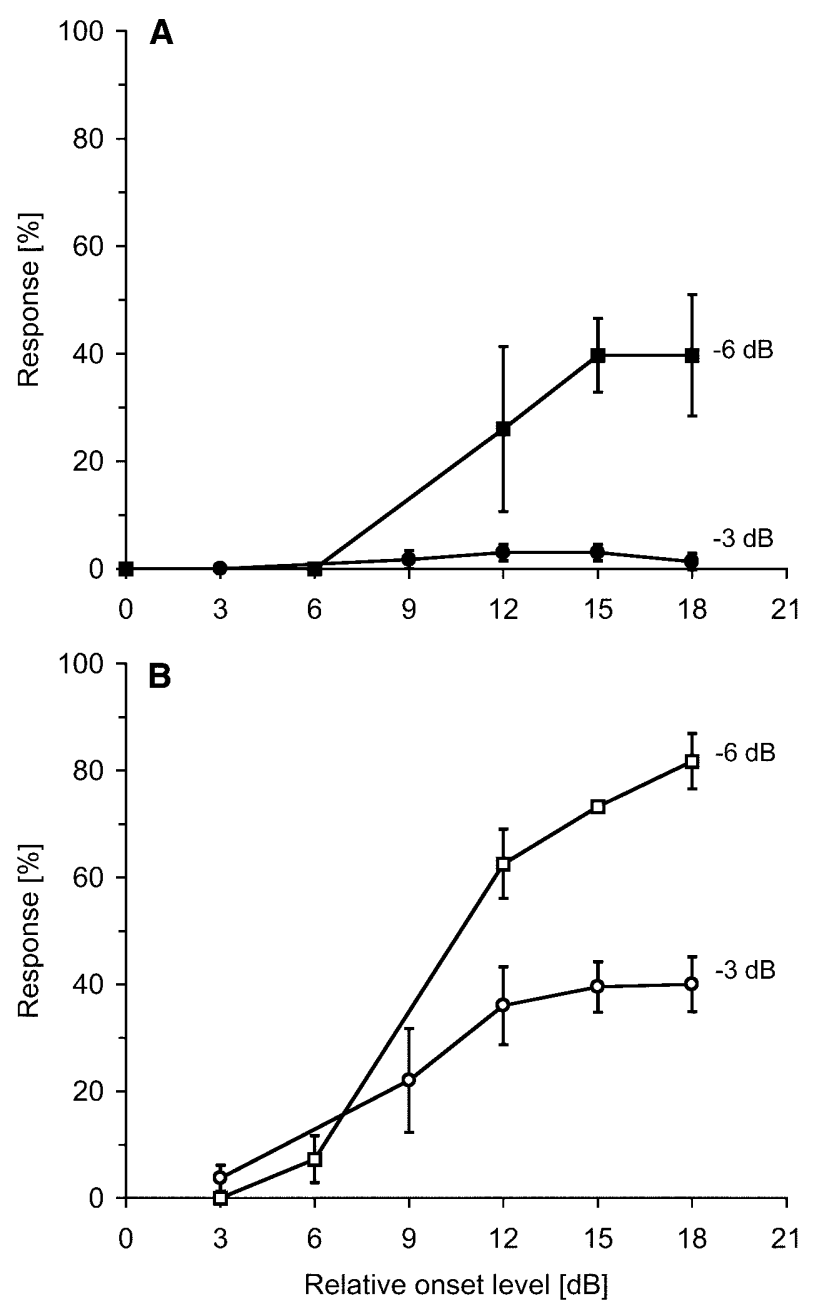

Fig. 3 The effect of increasing the relative offset level of the pauses (of the stimulus series in Fig. 2) from $-3 \mathrm{~dB}$ with respect to the end of the syllable to $-6 \mathrm{~dB}$ on the females that showed low (A, $n=3$, closed symbols) and intermediate (B, $n=4$, open symbols) mean response levels in the previous experiment (see Fig. 2). In both groups of females the increased attenuation during the pause drastically increased the response level (squares compared to circles)

of syllable onsets in the context of the noisy, impactfilled pauses. To test this, we played back a series of model songs in which the first $10 \mathrm{~ms}$ of each syllable were systematically raised in amplitude in steps of $3 \mathrm{~dB}$ over that of the noisy pauses (relative onset level varied: see Fig. 2). The relative offset level was $-3 \mathrm{~dB}$ and was held constant in this experiment.

In 8 out of 11 females tested, increasing the relative onset level resulted in an increase in response (Fig. 2): four showed a steep increase, saturating at about $80 \%$, while the response of the other four females saturated at intermediate levels of about $40 \%$. Three females did not respond at all, even when the relative onset level was increased to $24 \mathrm{~dB}$. The low response of these females was not due to lack of motivation, since they responded well to a standard 80/12 song model (a verse containing $80 \mathrm{~ms}$ rectangular white noise syllables separated by $12-$ ms-long silent pauses).
Syllable offset cues are not always necessary

Seven out of 11 females tested in the previous experiment showed either intermediate ( $n=4$ females) or very low $(n=3$ females) responses even at high values of the relative onset level. A possible reason for this was that these females may have required a relative offset level that was greater than $-3 \mathrm{~dB}$. That this argument was valid was revealed by the increased response of both groups (females showing intermediate and low responses) when re-tested with the same stimuli as above, but with the relative offset level increased to $-6 \mathrm{~dB}$ (Fig. 3).

To examine the role of the relative offset level, a series of model songs was constructed in which the relative offset level was varied systematically up to $-15 \mathrm{~dB}$ attenuation while keeping the relative onset level constant at $15 \mathrm{~dB}$. Of seven females tested, all showed an increase in response with increasing values of the relative offset level (Fig. 4). There was, however, a large inter-individual variation in the value of the relative offset level required for a half-maximal response, ranging from $0 \mathrm{~dB}$ (no difference in intensity between the end of the syllable and the pause) to $-12 \mathrm{~dB}$. Interestingly, the two females that required little or no intensity difference at offset in fact showed a decrease in response with lower sound levels in the pauses, i.e. they appeared to prefer 'noisy' pauses.

The observation that two females in the previous experiment responded in the absence of intensity differences at the syllable offset suggested that the presence of syllable offset cues may not always be necessary. To test this, we constructed song stimuli that lacked syllable offset cues: each stimulus consisted of a verse constructed by 32 repetitions of a noise 'syllable' ( $92 \mathrm{~ms}$ in length) with no pauses in between. The first $10 \mathrm{~ms}$ of each 'syllable' was raised in amplitude to mark the 'syllable' onset and the extent of the accentuation was varied from $0 \mathrm{~dB}$ to $21 \mathrm{~dB}$ in steps of $3 \mathrm{~dB}$ through the stimulus series (see Fig. 5). Of ten females tested, four showed very poor or no responses (Fig. 5) to all the test stimuli (they did, however, respond to a standard 80/12 song model with silent pauses). Interestingly, these four females had also been tested in the previous experiment, where they showed an increase in response with increasing relative offset level, i.e. they showed an absolute requirement for syllable offsets. Four females showed a narrowly 'tuned' response, i.e. they responded strongly only at certain values of syllable onset accentuation (Fig. 5). The remaining two females showed a steep increase in response as a function of increasing onset accentuation and continued to respond with high probability (Fig. 5), i.e. they showed a saturating response.

In order to test whether the requirement for syllable offsets was an intensity-dependent phenomenon, we played back the same stimulus series (illustrated in Fig. 5) to females at three different intensities $(58 \mathrm{~dB}$, $64 \mathrm{~dB}$ and $70 \mathrm{~dB}$ SPL). Female response profiles were consistent over a 12-dB range of intensites $(n=10$ females; responses of three individuals illustrated in Fig. 6). Females that required syllable offsets (example 
Fig. 4 The effect of varying the relative offset level. The graph shows the results of experiments in which the sound level of the pauses with respect to the syllable end (in a model song of 80-ms noise syllables and 12-ms pauses) was varied systematically in 3-dB steps. The relative onset level was kept constant at $15 \mathrm{~dB}$. Each line represents the response of one female ( $n=7$ females). The dotted line indicates $50 \%$ of the maximum response of each female

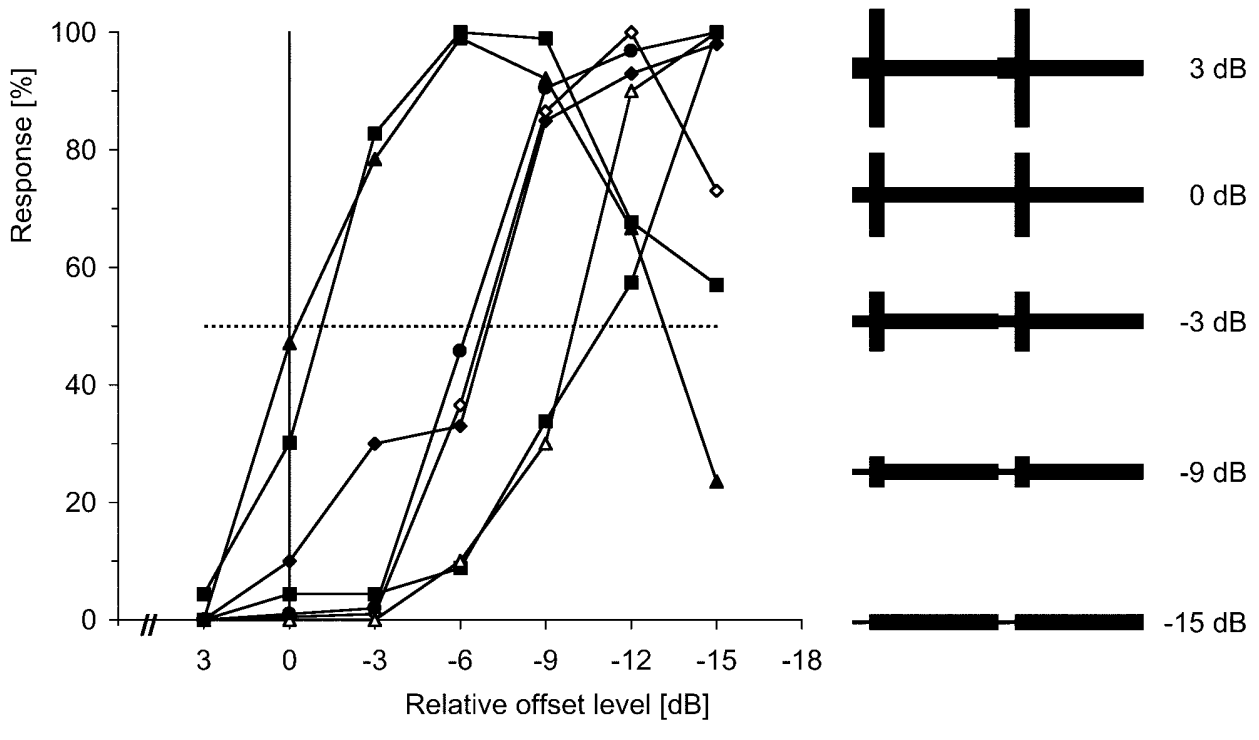

illustrated in Fig. 6, top) did not respond to song models lacking pauses at any playback intensity. Those showing a 'tuned' response (example illustrated in Fig. 6, middle) were also consistent across the different intensities of playback, as were the individuals that showed a 'saturating' response (example illustrated in Fig. 6, bottom).

The above results indicate that there are two types of females: those that require both onset and offset cues

Fig. 5 The effect of removing the syllable pauses. The graph shows the responses of females to a series of model songs lacking pauses. The stimuli consisted of 3-s-long verses, each constructed by the repetition of a 92-ms-long noise 'syllable' with no pauses (syllable period $=92 \mathrm{~ms}$ ). The first $10 \mathrm{~ms}$ of each 'syllable' was accentuated in amplitude over the rest of the noise stimulus (shown in the stimulus series on the right: the first increase was a step of $6 \mathrm{~dB}$, the succeeding steps being $3 \mathrm{~dB}$ each). Each line represents the response of one female ( $n=10$ females). The symbols represent females which did not respond (closed circles), females which showed a 'tuned' response (triangles) and a saturating response (squares) and those that require only onsets. This suggests that some females may not possess a functional syllable offset detection mechanism, the response being released by the onset detector alone.

\section{Onset and offset detection within individual females}

To examine the relation between onset and offset detection within individual females, we determined the range of effective onset accentuations and relative offset levels for individual females by simultaneously varying both from $0 \mathrm{~dB}$ to $18 \mathrm{~dB}$ through a stimulus series (see Fig. 7, bottom panel), which was then played back to 13 females. The values of onset accentuations and relative offset levels that resulted in a maximal response varied widely between females. Most females ( 7 out of 13) responded over a relatively broad range of onset accentuations and relative offset levels ( $-6 \mathrm{~dB}$ to $-18 \mathrm{~dB}$; Fig. 7B). A few females did,

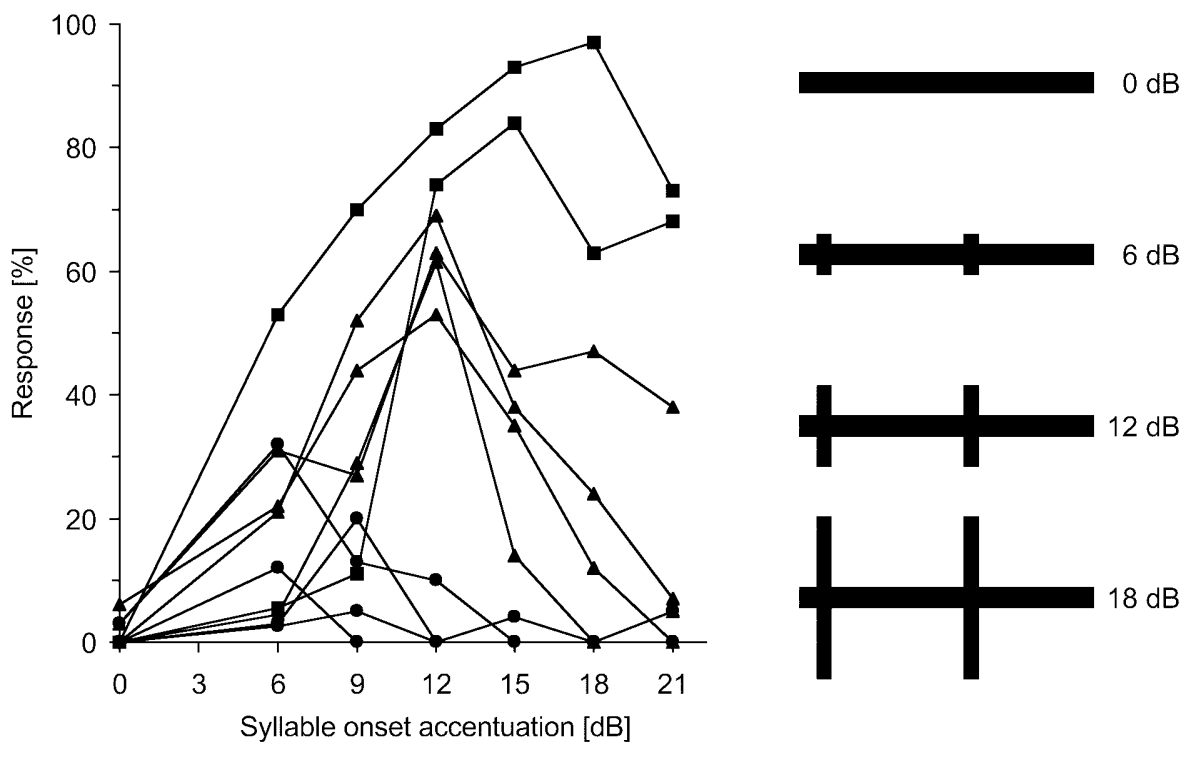



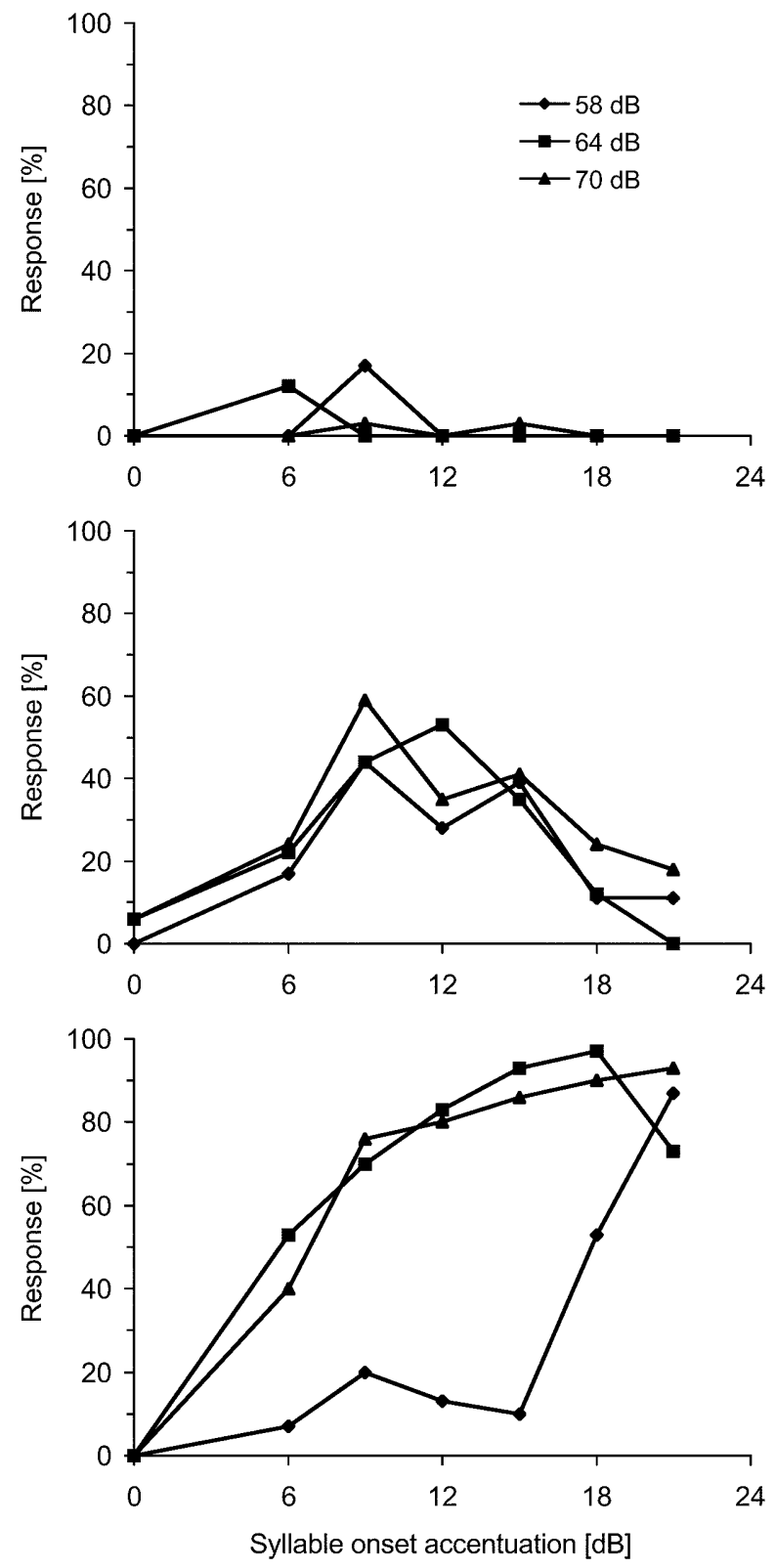

Fig. 6 The effect of intensity of playback on the response profile of individual females to song models lacking syllable pauses (stimuli illustrated in Fig. 5). Each graph illustrates the response of one female. Stimuli were played back at $58 \mathrm{db}, 64 \mathrm{db}$ and $70 \mathrm{~dB}$ SPL (for the non-amplitude-modulated part of the stimulus). Top: example of a female that showed no response in the absence of pauses. Middle: example of a female showing a 'tuned' response to the syllable onset accentuation. Bottom: example of a female showing a saturating response: the different response profile at $58 \mathrm{~dB}$ SPL may be due to a relatively high hearing threshold in this individual

however, need exceptionally high relative offset levels to evoke a response (Fig. 7C), whereas others responded in the absence of pauses (Fig. 7A). Interestingly, individuals that responded to stimuli without pauses typically needed higher onset accentuations (12-18 dB) and showed a decrease in response to high relative offset levels $(-12 \mathrm{~dB}$ and higher), preferring noisy pauses.
Do females that require no offset cues possess a functional offset detection mechanism?

We selected three females that responded to song models lacking syllable offset cues (noise stimuli with no pauses, as in Fig. 5, with 12-dB syllable accentuation). These females were then tested with a series of song models containing clear pauses, in which the pause duration was systematically varied between $0 \mathrm{~ms}$ and $50 \mathrm{~ms}$. The results (Fig. 8) showed that, in the presence of clear pauses, the response was limited to a relatively narrow range of pause durations. Thus, females that do not need syllable offset cues nevertheless appear to possess a functional pause detection mechanism that restricts the range of acceptable pause durations.

\section{Discussion}

Our data suggest that syllable onset and offset detection in Ch. biguttulus females are separate processes. Syllable onset cues (achieved either by the presence of a preceding pause or the accentuated beginning of a syllable, or both) were always necessary, whereas syllable offsets were not always required: some females responded to song models that lacked pauses. Further, the responses of females that did not require syllable offsets were nevertheless affected by the presence of offset cues. Firstly, when presented with stimuli containing clear pauses, they responded to a defined range of pause durations (5-30 ms: Fig. 8), which means that they were able to perceive offsets. Secondly, some of these females preferred 'noisy pauses' over clear pauses (Fig. 4 and Fig. 7, top).

Inter-individual variation in response profiles

Our experiments revealed a large variation between individuals with respect to the values of the relative onset and offset levels required for optimal responses. Although onsets were always necessary, the range of relative onset levels for a half-maximal response varied between $9 \mathrm{~dB}$ and $18 \mathrm{~dB}$ for different individuals. Offset detection was even more variable, the value of the relative offset level required for a half-maximal response varying between $0 \mathrm{~dB}$ and $-12 \mathrm{~dB}$. Thus, some females required large relative offset levels whereas others did not require any syllable offsets and responded to stimuli that completely lacked them. Ronacher and Krahe (1998) reported similar observations with respect to the recognition of female Ch. biguttulus song by conspecific males: some males did respond to female song models that lacked syllable pauses, provided that the syllable onsets were clearly marked by an accentuation in amplitude. Males also exhibited a large inter-individual variation in response to female song models that lacked syllable pauses (Ronacher and Krahe 1998). 
We believe that the variety of response profiles observed with respect to syllable onset and offset detection reflects the range of inter-individual phenotypic variation rather than the effects of age or temporary physiological status, for the following reasons: (1) whenever individuals were re-tested (two or three times) with the same stimulus series, their responses were consistent; and (2) there was no correlation of any response profile with age at testing. There was also no correlation between response profile and local area of collection, making it unlikely that genetic differences between local populations were responsible for the observed heterogeneity of responses.

\section{A model for syllable-pause detection}

We propose a simple model for the mechanism of syllable-pause detection in Ch. biguttulus (Fig. 9), based both on our present experiments and on previous work. We postulate the existence of two processes: syllable onset detection and the detection (and rejection) of unacceptably long pauses (greater than about $30 \mathrm{~ms}$ at $30^{\circ} \mathrm{C}$ ).

Onset detection would involve a neuronal unit that responds phasically to increases in intensity level (syllable onsets) and can be activated again only after a refractory period. One simple possibility would be that this neuronal unit is inhibited during the course of the syllable by delayed inhibition via a second input. The onset detector would be released from inhibition during the pause (because the decrease in intensity during a pause would cause a decrease in the activity of the inhibitory input), enabling it to respond to the next syllable onset.

The detection of long pauses may involve one or more units that are best activated by continuous noisy signals or those with a high duty cycle (above $80 \%$ ). The output of this unit would be ANDed with that of the syllable onset detector at the next level of processing.

The observed inter-individual variation in response profiles can be accounted for within the framework of the proposed model. Syllable onset cues are clearly necessary and the accentuated syllable onsets in male songs would provide the necessary increase in intensity over the noisy pauses required to activate the onset detector. The inter-individual variation in the optimal

Fig. 7A-C The range of effective syllable onset accentuations and relative offset levels in individual females. Syllable onset accentuation and relative offset levels were co-varied through the stimulus series. The three graphs from the top show the response profiles of three females, illustrating the variation between individuals that A respond in the absence of pauses, $\mathbf{B}$ respond over a wide range of relative onset and offset levels, and $\mathbf{C}$ require large relative offset levels. The stimuli are illustrated in the bottom panel. Each filled circle represents the percentage response (value shown below the circle) to a song model with a given combination of onset accentuation and relative offset level
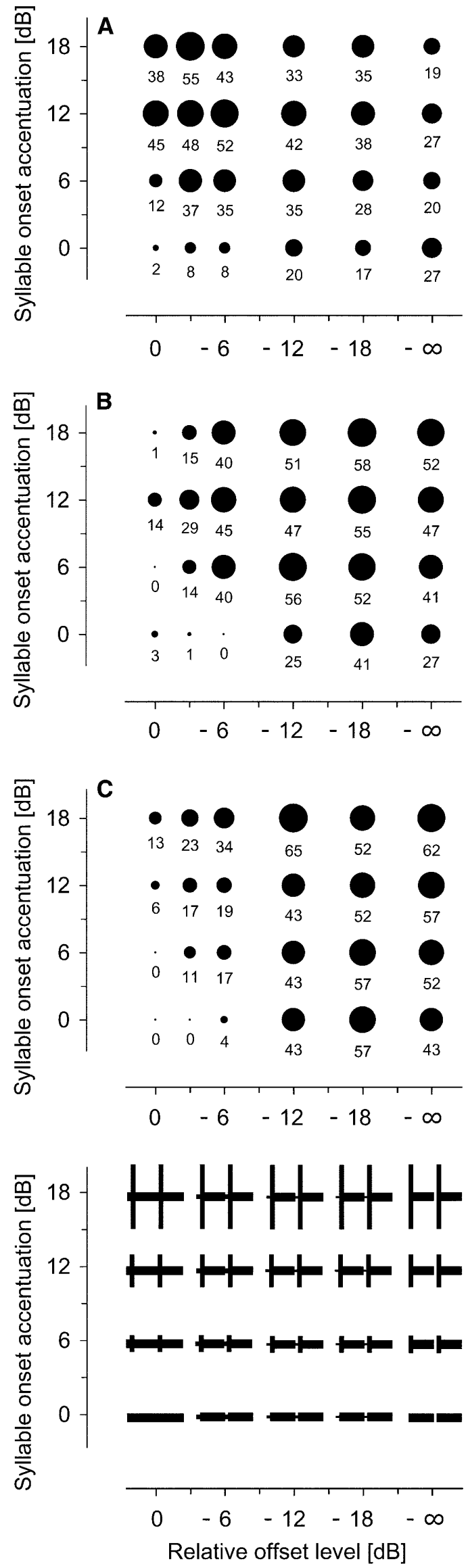

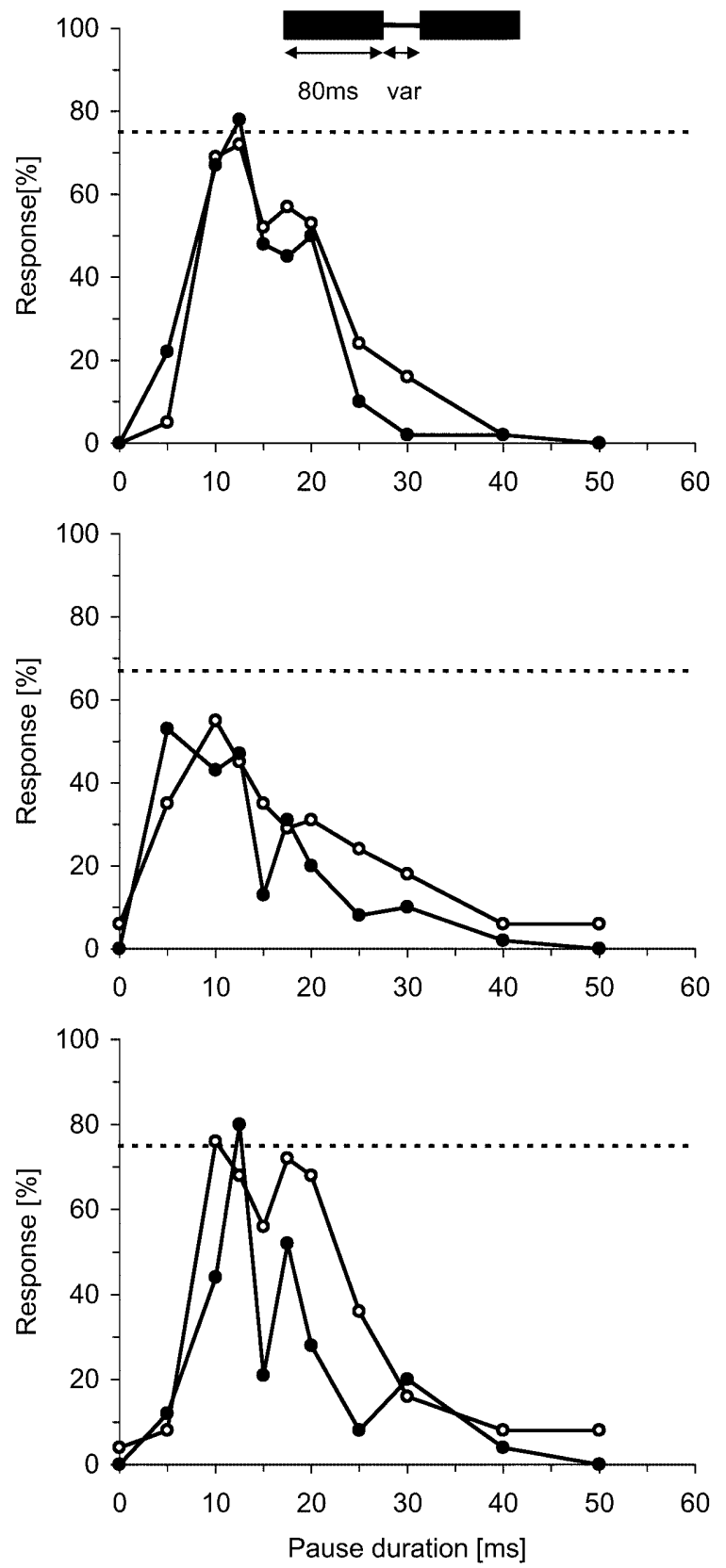

Fig. 8 The responses of individuals that did not require syllable offset cues to stimuli containing silent pauses. Each graph illustrates the response of an individual female to a series of song models (3-s-long verses, consisting of $80-\mathrm{ms}$ noise syllables separated by silent pauses) in which the pause duration was systematically varied. Stimuli were played back at $64 \mathrm{~dB}$ (open circles) and $76 \mathrm{~dB}$ SPL (closed circles). The dotted line indicates the response to a song pattern that lacked pauses (syllable onset accentuation $=12 \mathrm{~dB}$, played back at $64 \mathrm{~dB}$ SPL for the nonamplitude-modulated part of the stimulus)

syllable relative onset levels could be due to differences in the physiological properties of the onset detector between individuals.

The differences between females that required syllable offset cues and those that responded in the absence of pauses cannot be explained by the lack of offset detec- tion in the latter because females who did not require syllable offset cues nevertheless showed a sharply tuned response to pause durations in artificial stimuli with silent pauses (Fig. 8). Thus, these individuals must possess a pause detector that restricts the range of acceptable pause durations (the 'detector of too long pauses' in Fig. 9). We believe rather that the differences in response between females that require syllable offset cues and those that do not could be explained by inter-individual differences in the physiology of the inhibitory input to the onset detector. If this unit fires tonically during the syllable and shows slow adaptation, then the individual would require the decrease in intensity at offset in order to release the inhibition on the onset detector, which would thus not be enabled in the absence of syllable offset cues (see Fig. 9). On the other hand, a strongly adapting response of this unit during the course of the syllable would result in a release of inhibition on the onset detector even in the absence of syllable offset cues, and such individuals would respond positively in the absence of pauses (Fig. 9).

The preference for noisy pauses shown by some females may be due to a comparatively high level of activation of the second unit (which is best activated by uninterrupted noisy stimuli and responsible for rejection of long pauses) in these individuals.

Onset detection and the detection of long pauses appear to be separate, independently varying processes, accounting for the large variety of response profiles among individual females. The two extremes of the adapting properties of the inhibiting unit (Fig. 9) may account for the two 'types' of females found in the experiments.

\section{Neuronal correlates}

In a landmark study in the field cricket Gryllus bimaculatus, Schildberger (1984) demonstrated the existence of neurons in the brain that could serve as bandpass filters for syllable repetition rates. Similarly, in Ch. biguttulus, Ronacher and Stumpner (1988) and Stumpner et al. (1991) discovered single ascending neurons whose activity profiles provided good neuronal correlates of gap detection and syllable onset detection. Stumpner et al. (1991) examined the physiological properties of 16 ascending auditory interneurons of Ch. biguttulus in response to model song stimuli in an attempt to identify single neurons involved in specific aspects of temporal pattern processing.

One of the neurons, AN12, gave a highly phasic response at the start of each syllable over a range of intensities and may be a candidate for the syllable onset detector proposed in the above model. When tested with artificial song models containing silent pauses, the AN12 required a minimum pause duration of 5-8 $\mathrm{ms}$ in order to respond to syllable onsets, correlating well with the rising phase of the behavioural response curve (Stumpner et al. 1991). The decrease in behavioural response to song models containing silent pauses exceeding 
Fig. 9 A model for syllablepause detection in $\mathrm{Ch}$. biguttulus. The filled rectangular boxes represent song syllables and the gaps between them represent pauses. The traces above the song patterns portray the neuronal responses (action potentials) to these stimuli and refer to the outputs of the suggested model units or circuits (see text for details). Song patterns: $A$ standard $80 / 12$ stimulus, with $80-\mathrm{ms}$ syllables and $12-\mathrm{ms}$ silent pauses; $B$ continuous noise stimulus with accentuated 'syllable onsets' and no pauses; $C$ standard 80/12 stimulus with noisy pauses. Arrowheads indicate excitation and filled circles indicate inhibition

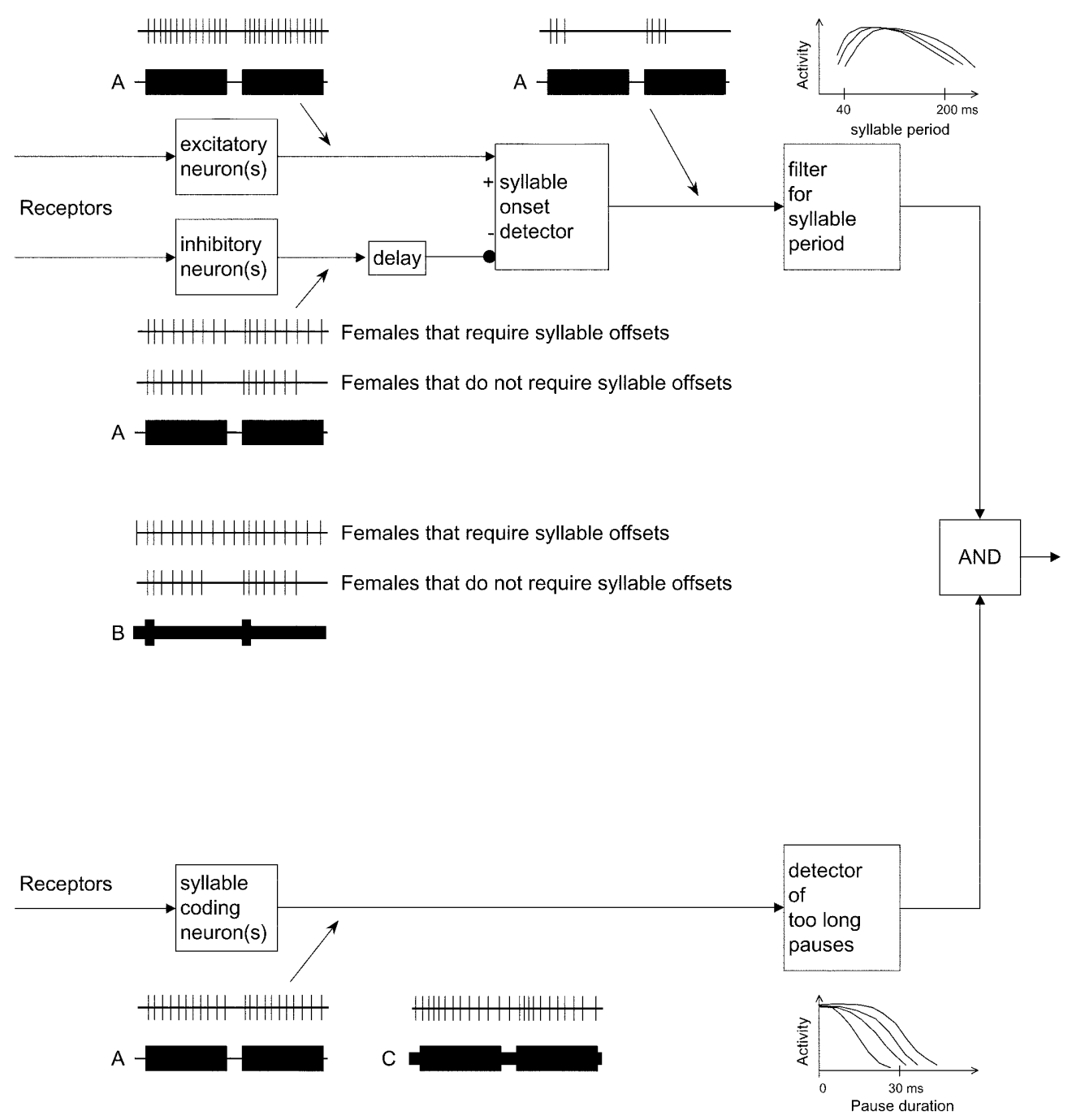

20-25 ms (Stumpner et al. 1991; von Helversen and von Helversen 1994) does not, however, have a neuronal correlate in terms of the response properties of the AN12 neuron.

The rejection of long pauses is probably mediated by a different neuronal unit. In principal, tonically active ascending neurons that roughly copy the stimulus pattern would be suited as an input of the second system assessing the duty cycle of the stimulus (e.g. the AN6, AN11, Stumpner et al. 1991). In particular, also the ascending neuron AN4 might be involved in that circuit: it is activated by continuous noise syllables but inhibited by gappy syllables. Such a response profile also correlates well with the rejection of syllables containing 2- to 3-ms gaps typical of one-legged males (Ronacher and Stumpner 1988; Stumpner et al. 1991).

Thus, the rising and falling phases of the behavioural response curve evoked by presentation of model song stimuli with varying pause durations (illustrated in Fig. 8) probably result from two separate, independent processes. The minimum pause duration is probably determined by the properties of the onset detector (Stumpner et al. 1991), which may require the pause in order to be released from inhibition. The rejection of long pauses is probably mediated by a second unit that is optimally excited by continuous, uninterrupted stimuli. The requirement for syllable offset cues is possibly determined by inter-individual differences in the physiological properties of the inhibitory input to the onset detector. Identified auditory interneurones have been shown to vary in their physiological properties both between and within individuals (Stumpner 1989): this physiological variability provides a plausible neuronal basis for the observed inter-individual differences in behavioural response profiles.

Further electrophysiological studies are needed at the level of the ascending auditory interneurons to compare their responses between individuals that show marked differences in the requirement for syllable offsets in behavioural experiments. In addition, the identification of neurons that are post-synaptic to the ascending interneurons in the brain, where the final stages of processing for pattern recognition take place (Bauer and von Helversen 1987), is necessary for a further understanding of the process of syllable-pause detection in this species. 
Acknowledgements We gratefully acknowledge Maria Bauer for her help in carrying out some of the experiments and her skilful preparation of the figures. Our thanks to Wolfram Schulze for his help with some of the experiments. We also thank Johannes Schul, Andreas Stumpner and Bernhard Ronacher for stimulating discussions. The experiments comply with the current German laws concerning animal care. This research project was funded in part by a postdoctoral fellowship awarded by the DFG to Rohini Balakrishnan and Dagmar von Helversen.

\section{References}

Alexander RD, Marshall DC, Cooley JR (1997) Evolutionary perspectives on insect mating. In: Choe JC, Crespi BJ (eds) The evolution of mating systems in insects and arachnids. Cambridge University Press, Cambridge, pp 4-31

Bauer M, Helversen O von (1987) Separate localisation of sound recognizing and sound producing neural mechanisms in a grasshopper. J Comp Physiol 161:95-101

Cade WH (1985) Insect mating and courtship behavior. In: Kerkut GA, Gilbert LI (eds) Comprehensive insect physiology biochemistry and pharmacology, vol 9. Pergamon Press; Oxford, pp 591-619

Elsner N (1974) Neuroethology of sound production in gomphocerine grasshoppers. I. Song patterns and stridulatory movements. J Comp Physiol 88:67-102

Elsner N (1983) A neuroethological approach to the phylogeny of leg stridulation in gomphocerine grasshoppers. In: Huber F, Markl H (eds) Neuroethology and behavioural physiology. Springer, Berlin Heidelberg New York, pp 54-68

Helversen D von (1972) Gesang des Maennchens und Lautschema des Weibchens bei der Feldheuschrecke Chorthippus biguttulus (Orthoptera, Acrididae). J Comp Physiol 81:381-422

Helversen D von, Helversen O von (1983) Species recognition and acoustic localization in an acridid grasshopper: a behavioral approach. In: Huber F, Markl H (eds) Neuroethology and behavioral physiology. Springer, Berlin Heidelberg New York, pp 95-107

Helversen D von, Helversen O von (1997) Recognition of sex in the acoustic communication of the grasshopper Chorthippus biguttulus (Orthoptera, Acrididae). J Comp Physiol 180: 373-386
Helversen D von, Helversen O von (1998) Acoustic pattern recognition in a grasshopper: processing in the time or frequency domain? Biol Cybern 79:467-476

Helversen O von, Elsner N (1977) The stridulatory movements of acridid grasshoppers recorded with an opto-electronic device. J Comp Physiol 122:333-340

Helversen O von, Helversen D von (1994) Forces driving coevolution of song and song recognition in grasshoppers. In: Schildberger K, Elsner N (eds) Neural basis of behavioural adaptations. Fortschr Zool 39:253-284

Hennig RM, Weber T (1997) Filtering of temporal parameters of the calling song by cricket females of two closely related species: a behavioral analysis. J Comp Physiol A 180:621-630

Michelsen A, Larsen ON, Surlykke A (1985) Auditory processing of temporal cues in insect songs: frequency domain or time domain? In: Michelsen A (ed) Time resolution in auditory systems. Springer, Berlin Heidelberg New York

Pollack GS (1998) Neural processing of acoustic signals. In: Hoy RR, Popper AN, Fay RR (eds) Comparative hearing: insects. Springer handbook of auditory research, vol 10. Springer, Berlin Heidelberg New York, pp 139-196

Ronacher B, Krahe R (1998) Song recognition in the grasshopper Chorthippus biguttulus is not impaired by shortening song signals: implications for neuronal encoding. J Comp Physiol A 183:729-735

Ronacher B, Stumpner A (1988) Filtering of behaviourally relevant temporal parameters of a grasshopper's song by an auditory interneuron. J Comp Physiol 163:517-523

Schul J (1998) Song recognition by temporal cues in a group of closely related bushcricket species (genus Tettigonia). J Comp Physiol A 183:401-410

Schildberger K (1984) Temporal selectivity of identified auditory neurons in the crickets brain. J Comp Physiol A 155:171-185

Stumpner A (1989) Physiological variability of auditory neurones in a grasshopper. Comparison of twin cells and mirror-image cells. Naturwissenschaften 76:427-429

Stumpner A, Ronacher B, Helversen O von (1991) Auditory interneurones in the metathoracic ganglion of the grasshopper Chorthippus biguttulus. II. Processing of temporal patterns of the song of the male. J Exp Biol 158:411-430

Weber T, Thorson J (1989) Phonotactic behavior of walking crickets. In: Huber F, Moore TE, Loher W (eds) Cricket behavior and neurobiology. Cornell University Press, Ithaca, NY, pp 310-339 